\title{
CAN NOVICE DRIVERS RECOGNIZE FORESHADOWING RISKS AS EASILY AS EXPERIENCED DRIVERS?
}

\author{
Lisandra Garay-Vega ${ }^{1}$, Donald L. Fisher ${ }^{2}$ \\ Human Performance Laboratory \\ ${ }^{1}$ Civil and Environmental Engineering \\ ${ }^{2}$ Mechanical and Industrial Engineering \\ University of Massachusetts, Amherst \\ Amherst, Massachusetts, USA \\ E-mail: lisandra@acad.umass.edu
}

\begin{abstract}
Summary: Novice drivers (16 and 17 years old) are almost ten times more likely to be involved in motor vehicle fatalities as adults 45-55 (NHTSA 2002). Besides traffic signs and other traffic control devices, there are many cues that help drivers further predict the presence of a potential risk in the driving environment. These cues are called foreshadowing elements (e.g., a pedestrian walking towards a crosswalk). It was hypothesized that given that younger adults have much less experience on the roads, it is more difficult for them to predict where potential cues might be positioned when foreshadowing elements are not present. However, in the presence of foreshadowing elements it was predicted that novice drivers should recognize risks as well as more experienced drivers. This research uses eye movement data gathered on a driving simulator to evaluate the use and effectiveness of the foreshadowing elements by novice and experienced drivers as predictors of areas in a scenario where risks may materialize. The research has potential implications for the sorts of instructional programs that might be developed for novice drivers.
\end{abstract}

\section{INTRODUCTION}

Several studies have been completed describing how glance behavior changes with driving experience. As drivers gain experience, they get better at scanning the roadway environment while ignoring irrelevant information. Eye movements change from narrow scanning and looking just ahead of the vehicle to sampling more widely as drivers' experience increases (Mourant et al., 1972). With experience, drivers develop automatic routines to help them maintain their lateral position, thereby freeing up resources that can then be devoted to scanning the roadway environment and acquiring information from farther ahead (Summala et. al., 1996). For this reason, experienced drivers may anticipate where potential cues may be positioned even when elements that foreshadow risks, such as conflicting traffic or pedestrian activity, are not present.

Since lack of experience influences the visual scanning pattern and, in particular, those areas of the roadway upon which a driver is fixating, studying eye-fixations should provide an understanding of pre-crash behavior and crash prevention. Indeed, recent studies suggest that this is the case. Novice, younger and older drivers' eye fixations were monitored as they drove through risky scenarios (Fisher et al., 2005; Pradhan et al., in press). In certain scenarios the novice drivers were some ten times less likely to fixate on the relevant areas of risk than were the more experience drivers. For example, consider the scenario in Figure 1 ("Truck in front of a crosswalk" scenario). Imagine for the moment that the pedestrian was not present in the 
crosswalk and that the participant driver (green car) is following another lead driver (red car). The participant driver should look to the right as he or she crosses in front of the truck parked on the right (gray vehicle) since it could be obscuring the participant driver's view of a pedestrian in the crosswalk. In fact, when this was measured, novice drivers looked to the right only $5 \%$ of the time, whereas more experienced drivers looked to the right $50 \%$ of the time.

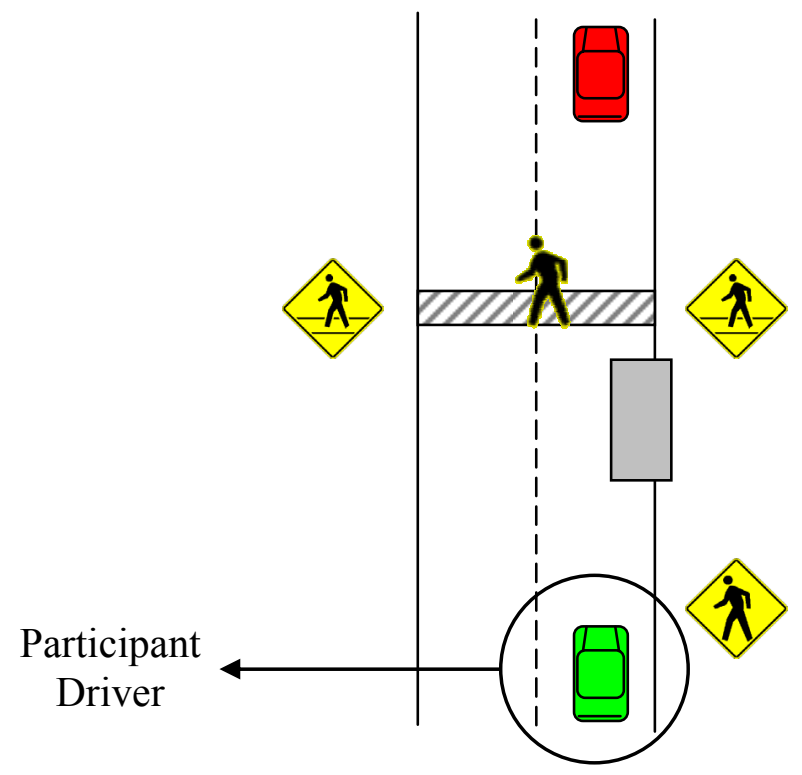

Figure 1. "Truck in front of a crosswalk" scenario

Two questions still need to be answered. First, in the studies run to date, no foreshadowing elements have been present (e.g., a pedestrian was not present in the cross walk and visible to the participant driver as he or she approached the cross walk). Given the clear relation between the foreshadowing element (e.g., a pedestrian visible to the participant driver) and the potential risk (a pedestrian present in the crosswalk, but hidden by the parked vehicle), one can reasonably ask whether novice drivers will perform as well as experienced drivers when the foreshadowing element is present. Second, and related to the above, it needs to be further determined how likely novice and experienced drivers are to recognize a risk, given that they fixate the foreshadowing element. Novice drivers could be less likely to recognize the risk, even when a foreshadowing element is present, simply because they fixate the foreshadowing element less often.

This paper describes a study that utilizes an advanced driving simulator and a head mounted eyetracker to determine if novice drivers recognize foreshadowed risks as often as experienced drivers and, if not, whether they recognize them less often simply because they fixate the foreshadowing element less often.

\section{METHOD}

Based on the results of a crash data analysis and previous research on driving performance among novice, inexperienced drivers, a virtual environment was developed for use on the driving simulator at the University of Massachusetts, Amherst. Both novice and experienced drivers were asked to navigate scenarios, which contained potential risks to which attention should be 
given. Each of the scenarios contained an advance warning sign and an area where a potential risk could emerge.

For example, consider again the "Truck in front of a crosswalk" scenario. Here, assume that the foreshadowing element was present, as illustrated in Figure 1. A pedestrian (foreshadowing element) was crossing in front of the truck as the driver approached the crosswalk (but long before the driver actually arrived at the crosswalk). It was inferred that the driver did not recognize the potential risk presented by a pedestrian emerging from in front of the parked truck if he or she did not look to the right as he or she passed the vehicle (no pedestrian ever emerged as the driver passed the truck). The same scenario was repeated when the foreshadowing element was not obvious (i.e., when a pedestrian did not cross in front of the truck within sight distance of the driver). Eye movement data was recorded in real time.

In general, it was hypothesized that when the only cues available were ones that were useful to drivers with some experience, novice drivers would be less likely to predict risky events presented in the scenario when compared with experienced drivers. However, when cues (foreshadowing elements) were available that novice as well as experienced drivers should recognize as predictive of risks, then novice drivers would be as likely to predict risky events as more experienced drivers.

\section{Participant Drivers}

A total of 48 drivers participated in the experiment. Twenty-four were novice drivers, between 16 and 17 years old, with one to six months of driving experience, and 24 were experienced drivers between 40 and 50 years old with more than 20 years of driving experience. The average age of each group was 16.5 and 44.7 years, respectively. Drivers were screened for age and allowed to participate only if they had a valid driver's license or learner permit. The recruiting process for drivers was conducted in the local area. Flyers were posted around the University of Massachusetts at Amherst, Amherst's high schools, local newspapers and local businesses in the town of Amherst.

\section{Equipment}

The study was performed on a fixed-based driving simulator in the Human Performance Laboratory at the University of Massachusetts. The simulator is an actual Saturn sedan, illustrated in Figure 2. The visual world is displayed on three screens, allowing 150 degrees of vision in the horizontal direction and 30 degrees in the vertical direction. The images can be displayed with a resolution as high as 1024 X 768 dpi in each screen, with a refresh rate of 60 Hz. The simulator also employs a surround sound audio system.

A head mounted eye tracker, illustrated in Figure 3, was also used. The unit is capable of sampling the position of the eye at $200 \mathrm{~Hz}$ and allows the drivers' head a full range of motion. The system overlaps the eye position on a video of the scene that is being viewed by a person wearing the eye tracker, converting eye position to an external point of gaze. This instrument was used to determine whether drivers identified a risk in the road ahead, and in particular, if they fixate specific areas in each scenario. 


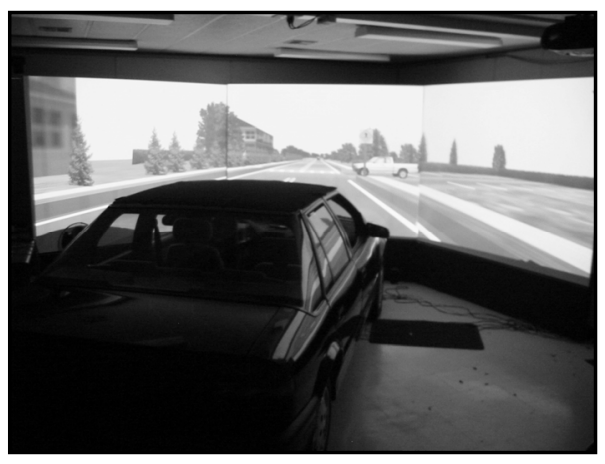

Figure 2. Driving Simulator, Human Performance Laboratory, UMass

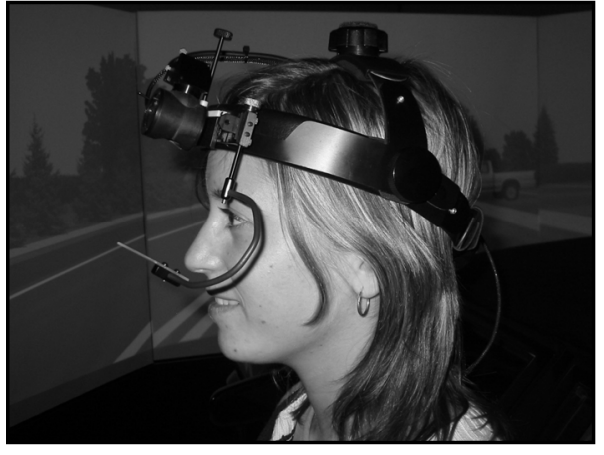

Figure 3. Head-mounted eye-tracker

\section{Design}

Nine scenarios were developed to use in the driving simulator, three of them with an element present which foreshadowed a potential risk. The three scenarios are displayed in, respectively, Figures 4-6 in the results section. The independent variables are driving experience (novice vs. experienced) and foreshadowing (present or absent).

\section{Dependent Variables and Data Analysis}

The eye tracker allowed researchers to determine whether a driver fixated specific areas in each scenario. Reference points delimited each area. If the driver fixated within the area, the driver was considered to have looked at the cues and/or at risky situations presented along the road. It was assumed that the driver predicted the risk if he or she fixated the critical area where a risk would emerge. If the driver fixated the critical area, a hit or a correct response was recorded $($ value $=1)$, otherwise a miss was recorded (value $=0)$.

In order to determine the importance of the foreshadowing element in the recognition of risk, an analysis was then performed to evaluate whether drivers: (1) looked at the foreshadowing element and risk, (2) looked at the foreshadowing element, but did not look at risk, (3) did not look at the foreshadowing element, but did look at risk, or, (4) did not look at the foreshadowing element or risk. These results were used to compute the conditional probability that drivers recognized a risk, given that they fixated the foreshadowing element.

\section{RESULTS}

Over all three scenarios averaged across both cases where the foreshadowing element was and was not present, $73.6 \%$ of the experienced drivers but only $38.2 \%$ of the novice drivers recognized risks. This was not just due to the differences in the percentage of experienced drivers recognizing risks when no foreshadowing was present. It is true that there were large differences in this case. In particular, over the three scenarios where the foreshadowing element was not present, 68.1 percent of the experienced drivers recognized risks and 33.3 percent of the novice drivers recognized risks. However, these differences were almost as large when the 
foreshadowing element was present. In particular, 79.2 percent of the experienced drivers recognized the risks when the foreshadowing element was present whereas, only 43.1 percent of the novice drivers recognized the risks. This difference varied from one scenario to the next. For example, in the scenario described above (Scenario 1: Truck in front of crosswalk), 79.2 percent of the experienced drivers recognized the risk while only 58.5 percent of the novice drivers recognized the risk (foreshadowing element present).

In order to determine the importance of the foreshadowing element in the recognition of risk, an analysis was performed to evaluate whether drivers: (1) looked at the foreshadowing element and risk, (2) looked at the foreshadowing element, but did not look at risk, (3) did not look at the foreshadowing element, but did look at risk, or (4) did not look at the foreshadowing element or risk. Among experienced drivers, in Scenario 1 (Figure 4) 75 percent looked at the foreshadowing elements and recognized the risk, while only 45.8 percent of the novice drivers looked at the foreshadowing elements and recognized the risk (Table 1). Similar differences hold for the other two scenarios (Figures 5 and 6).

More importantly, these results were used to compute the conditional probability that drivers recognized a risk, given that they fixated the foreshadowing element. Overall, $85.0 \%$ of the experienced drivers recognized a risk given that they fixated the foreshadowing element whereas only $47.5 \%$ of the novice drivers recognized a risk, given that they fixate the foreshadowing elements. These percentages are significantly different from one another, as is the percentage of novice drivers recognizing the risk given that they fixated the foreshadowing element (47.5\%), and the percentage of drivers recognizing the risk, given that they did not fixate the foreshadowing element (33.7\%). For experienced drivers in Scenario 1, 86\% of this group recognized the risk, given that they looked at the foreshadowing element (Table 2). However, for novice drivers, only $61 \%$ of this group recognized the risk, given that they looked at the foreshadowing element. Similar differences hold for the other two scenarios.

Table 1. Marginal Probabilities

\begin{tabular}{|l|c|c|c|c|c|c|}
\hline \multirow{2}{*}{} & \multicolumn{5}{|c|}{ Scenario } \\
\cline { 2 - 7 } & $\mathbf{1}$ & $\mathbf{2}$ & $\mathbf{3}$ & $\mathbf{1}$ & $\mathbf{2}$ & $\mathbf{3}$ \\
\cline { 2 - 7 } & \multicolumn{3}{|c|}{ Experienced } & \multicolumn{4}{c|}{ Novice } \\
\hline Looked at foreshadowed element and risk & .75 & .792 & .375 & .458 & .458 & .083 \\
\hline Looked at foreshadowed element did not look at risk & .125 & .000 & .167 & .292 & .250 & .417 \\
\hline Did not look at foreshadowed element but looked at risk & .042 & .208 & .208 & .125 & .125 & .042 \\
\hline Did not look at foreshadowed element nor risk & .083 & .000 & .250 & .125 & .167 & .458 \\
\hline
\end{tabular}

Table 2. Conditional Probabilities

\begin{tabular}{|l|c|c|c|c|c|c|}
\hline & \multicolumn{5}{|c|}{ Scenario } \\
\cline { 2 - 6 } & \multicolumn{1}{|c|}{$\mathbf{1}$} & $\mathbf{2}$ & $\mathbf{3}$ & $\mathbf{1}$ & $\mathbf{2}$ & $\mathbf{3}$ \\
\cline { 2 - 6 } & \multicolumn{3}{|c|}{ Experienced } & \multicolumn{3}{|c|}{ Novice } \\
\hline Prob. look at risk given that the driver looked at foreshadowed & 0.86 & 1.00 & 0.69 & 0.61 & 0.65 & 0.17 \\
\hline Prob. looked risk given that the driver did NOT look at foreshadowed & 0.34 & 1.00 & 0.45 & 0.50 & 0.43 & 0.08 \\
\hline
\end{tabular}




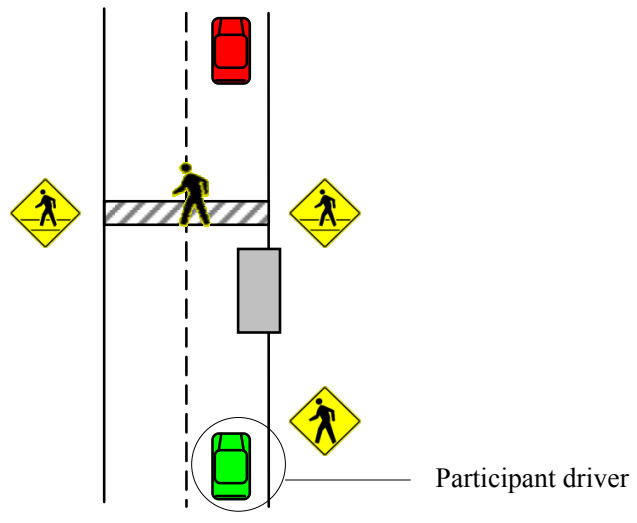

- Advance Cue: Pedestrian Ahead Sign

- Foreshadowing Element: Pedestrian

口 Critical Are: Sidewalk

Figure 4. Scenario 1, "Truck in Front of Crosswalk"

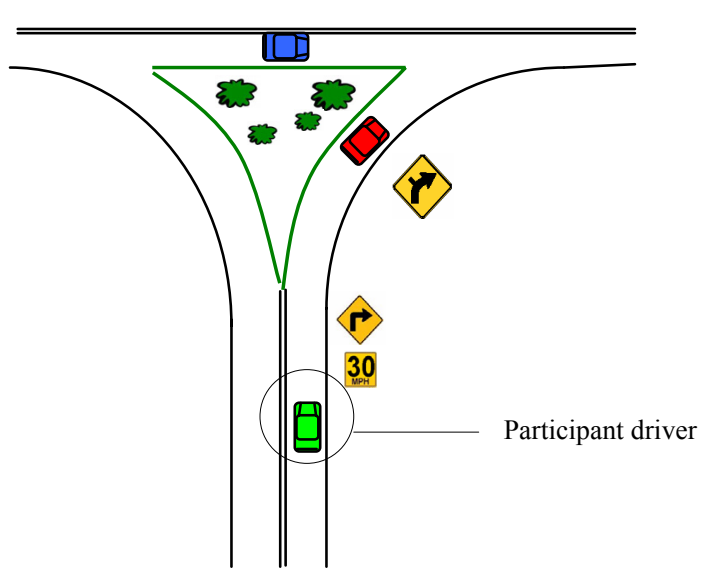

口 Advance Cue: Intersection/Curve Sign

- Foreshadowing Element: motorcycle traveling in major roadway

- Critical Are: Merging area

Figure 5. Scenario 2, "Yield to Traffic"

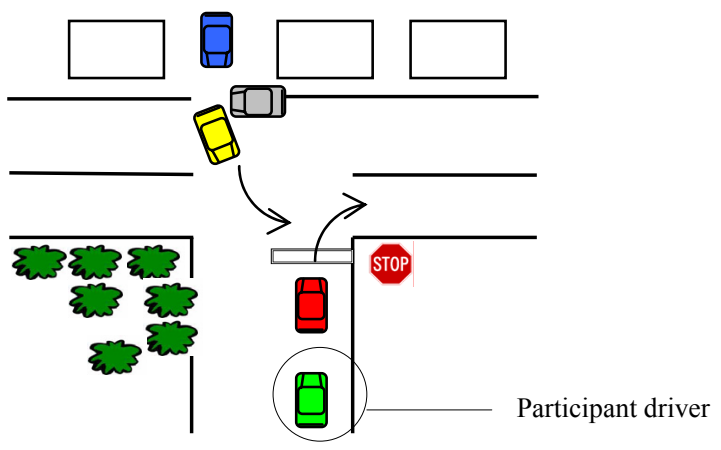
- Advance Cue: Vehicle from driveway
- Foreshadowing Element: Vehicle at driveway
- Critical Area: Driveway

Figure 6. Scenario 3, "Turning Vehicle at Two-Way Stop Control Intersection" 


\section{CONCLUSIONS}

In general, when novice drivers saw the foreshadowing element, they were almost two times as likely to recognize a risk as when the foreshadowing element was not present. However, the foreshadowing element by itself was not enough to equalize novice and experienced drivers' behavior. Specifically, the precentage of experienced drivers recognizing the risk, given that they saw the foreshadowing element (85\%) was significantly larger than the percentage of novice drivers recognizing the risk, given that they too saw the foreshadowing element $(47 \%)$. This was particularly true in situations involving pedestrians. A similar trend was observed in situations involving traffic signs and conflicting traffic. Performance among the novice drivers was even worse when a foreshadowing element was not present. Only $33 \%$ of the novice drivers recognized risks in these situations. The evidence continues to point to the need for training in the relevant visual scanning and risk-prediction skills. Specifically, training programs should be devised that single out the importance of the risks in situations in which a foreshadowing element is not present, since in those situations novice drivers are more than twice as likely to miss the potential risk.

\section{REFERENCES}

Fisher, D. L., Narayanaan, V., Pradhan, A. and Pollatsek, A. (2004). The Use of Eye Movements to Evaluate the Effect of PC-Based Risk Awareness Training on an Advanced Driving Simulator. Proceedings of the Human Factors and Ergonomics Society's Annual Meeting, New Orleans.

Mourant, R. R.and Rockwell, T.H. (1972). Strategies of visual search by novice and experienced drivers. Human Factors, 14(4): 325-335.

National Highway Traffic Safety Administration. (2002). Traffic Safety Facts 2001. U.S. Department of Transportation.

Pradhan, A. K., Hammel, K. R., DeRamus, R., Pollatsek, A., Noyce, D. A. and Fisher, D. L. (In press). The Use of Eye Movements to Evaluate the Effects of Driver Age on Risk Perception in an Advanced Driving Simulator. Human Factors.

Summala, H., Nieminen, T. and Punto, M. (1996). Maintaining lane position with peripheral vision during in-vehicle tasks. Human Factors, 38: 442-451. 